\title{
THE DIVERGENCE OF SEQUENCES OF POLYNO- MIALS INTERPOLATING IN ROOTS OF UNITY
}

\author{
BY J. L. WALSH
}

Polynomials defined by the property of interpolation to a given function in the roots of unity have been studied by Méray, Runge, Fejér, and the present writer, ${ }^{*}$ and are of considerable interest. Thus the following theorem has been established, except for the last sentence.

TheOREm. Let the function $f(z)$ be single-valued and analytic throughout the interior of the circle $|z|=\rho>1$, but not singlevalued and analytic throughout the interior of any larger concentric circle. Let $p_{n}(z)$ be the unique polynomial $\uparrow$ of degree $n$ which interpolates to $f(z)$ in all the $(n+1)$ st roots of unity. Then the sequence $p_{n}(z)$ converges to $f(z)$ throughout the region $|z|<\rho$, uniformly on any closed point set interior to that region. The sequence $p_{n}(z)$ diverges at every point exterior to the circle $|z|=\rho$.

Hitherto it has been known $\ddagger$ merely that the sequence $p_{n}(z)$ diverges at every point of the region $\rho<|z|<\rho^{2}$; it is the object of the present note to establish divergence at every point exterior to the circle $|z|=\rho$.

In our proof it is convenient to have for reference several easily proved lemmas.

LEMMA 1. The relations

$$
\varlimsup_{n \rightarrow \infty}\left|A_{n}\right|^{1 / n}=q, \quad \varlimsup_{n \rightarrow \infty}\left|B_{n}\right|^{1 / n}=q_{1} \leqq q
$$

imply

$$
\varlimsup_{n \rightarrow \infty}\left|A_{n}+B_{n}\right|^{1 / n} \leqq q .
$$

* See, for instance, Walsh, Interpolation and Approximation, Colloquium Publications of this Society, vol. 20,1935, $\$ 7.1,7.10$.

$\dagger$ A polynomial of degree $n$ is an expression of the form $a_{0} z^{n}+a_{1} z^{n-1}+\cdots$ $+a_{n}$; we do not assume $a_{0} \neq 0$.

$\ddagger$ Walsh, op. cit., p. 154. 
Let $\epsilon>0$ be arbitrary. From equations (1) we have for $n$ sufficiently large

$$
\left|A_{n}\right| \leqq(q+\epsilon)^{n}, \quad\left|B_{n}\right| \leqq\left(q_{1}+\epsilon\right)^{n} \leqq(q+\epsilon)^{n} .
$$

Thus we have

$$
\left|A_{n}+B_{n}\right| \leqq 2(q+\epsilon)^{n}, \quad \varlimsup_{n \rightarrow \infty}\left|A_{n}+B_{n}\right|^{1 / n} \leqq q+\epsilon .
$$

When $\epsilon$ is allowed to approach zero, inequality (2) follows.

LEMma 2. The relations

$$
\varlimsup_{n \rightarrow \infty}\left|C_{n}\right|^{1 / n}=q, \quad \varlimsup_{n \rightarrow \infty}\left|D_{n}\right|^{1 / n}=q_{1}<q
$$

imply

$$
\varlimsup_{n \rightarrow \infty}\left|C_{n}+D_{n}\right|^{1 / n}=q .
$$

From Lemma 1 we conclude that the left-hand member of (4) is not greater than $q$. But

$$
\varlimsup_{n \rightarrow \infty}\left|C_{n}+D_{n}\right|^{1 / n}=q_{2}<q
$$

is impossible, for by (5) and the second of relations (3) we should have by setting $A_{n}=C_{n}+D_{n}, B_{n}=-D_{n}$ in Lemma 1

$$
\varlimsup_{n \rightarrow \infty}\left|C_{n}\right|^{1 / n} \leqq\left[\text { larger of } q_{1} \text { and } q_{2}\right]<q,
$$

in contradiction to (3).

Lemma 3. Let $s_{n}(z)$ denote the sum of the first $n+1$ terms of the series $\sum_{n=0}^{\infty} a_{n} z^{n}$. Let us suppose

$$
\varlimsup_{n \rightarrow \infty}\left|a_{n}\right|^{1 / n}=A>0 .
$$

Then we have for $|z| \geqq 1 / A$

$$
\varlimsup_{n \rightarrow \infty}\left|s_{n}(z)\right|^{1 / n}=A|z| \text {. }
$$

Let $\epsilon>0$ be arbitrary. For $n$ sufficiently large we have $\left|a_{n}\right|<(A+\epsilon)^{n}$, so the sequence $\left|a_{n}\right| /(A+\epsilon)^{n}$ has a finite upper bound $M$ : 


$$
\begin{aligned}
&\left|a_{n}\right| \leqq M(A+\epsilon)^{n} \\
&\left|s_{N}(z)\right| \leqq M \sum_{n=0}^{N}(A+\epsilon)^{n}|z|^{n}=M \frac{(A+\epsilon)^{N+1}|z|^{N+1}-1}{(A+\epsilon)|z|-1}, \\
& \varlimsup_{N \rightarrow \infty}\left|s_{N}(z)\right|^{1 / N} \leqq(A+\epsilon)|z| ;
\end{aligned}
$$

allowing $\epsilon$ to approach zero then yields the relation

$$
\varlimsup_{n \rightarrow \infty}\left|s_{n}(z)\right|^{1 / n} \leqq A|z| \text {. }
$$

Let us now assume the inequality to hold in (8):

$$
\varlimsup_{n \rightarrow \infty}\left|s_{n}(z)\right|^{1 / n}<Q<A|z|
$$

we shall reach a contradiction. For $n$ sufficiently large we have

$$
\begin{gathered}
\left|s_{n-1}(z)\right|<Q^{n-1}, \quad\left|s_{n}(z)\right|<Q^{n}, \\
\left|s_{n}(z)-s_{n-1}(z)\right|=\left|a_{n} z^{n}\right|<Q^{n}(1+1 / Q), \\
\left|a_{n}\right|<Q^{n}(1+1 / Q) /|z|^{n}, \\
\overline{\lim }_{n \rightarrow \infty}\left|a_{n}\right|^{1 / n} \leqq Q /|z|<A,
\end{gathered}
$$

which contradicts (6). Equation (7) is established.

It is worth remarking that equation (7) may fail if the requirement $|z| \geqq 1 / A$ is omitted, as is illustrated by the series $\sum_{n=0}^{\infty} z^{n}$.

We are now in a position to establish the theorem. For the polynomials $p_{n}(z)$ already defined we have the interpolation formula

(9) $f(z)-p_{n}(z)=\frac{1}{2 \pi i} \int_{C_{R}} \frac{z^{n+1}-1}{t^{n+1}-1} \frac{f(t) d t}{t-z}, \quad\left(z\right.$ interior to $\left.C_{R}\right)$,

where $C_{R}$ denotes the circle $|z|=R, 1<R<\rho$. If $P_{n}(z)$ denotes the sum of the first $n+1$ terms of the Taylor development of $f(z)$ about the origin, then $P_{n}(z)$ is also the polynomial of degree $n$ which interpolates to $f(z)$ in the origin counted of multiplicity $n+1$, so we have

(10) $f(z)-P_{n}(z)=\frac{1}{2 \pi i} \int_{C_{R}} \frac{z^{n+1}}{t^{n+1}} \frac{f(t) d t}{t-z}, \quad\left(z\right.$ interior to $\left.C_{R}\right)$. 
Subtraction of (10) from (9) member by member yields the equation

$$
P_{n}(z)-p_{n}(z)=\frac{1}{2 \pi i} \int_{C_{R}} \frac{z^{n+1}-t^{n+1}}{t^{n+1}\left(t^{n+1}-1\right)} \frac{f(t) d t}{t-z} .
$$

Equation (11) is thus established for $z$ interior to $C_{R}$. But when the common factor $t-z$ is cancelled from numerator and denominator in the integrand in (11), it is seen that that integrand considered as a function of $z$ has no singularity for any (finite) value of $z$; hence the right-hand member of (11) represents a function of $z$ analytic for all finite values of $z$; the two members of (11) are equal for $z$ interior to $C_{R}$, so equation (11) is valid for all finite values of $z$.

For any finite value of $z$ exterior to $|z|=\rho$ we read off directly from (11)

$$
\varlimsup_{n \rightarrow \infty}\left|P_{n}(z)-p_{n}(z)\right|^{1 / n} \leqq|z| / R^{2} .
$$

But $R$ can be allowed to approach $\rho$, whence the inequality

$$
\varlimsup_{n \rightarrow \infty}\left|P_{n}(z)-p_{n}(z)\right|^{1 / n} \leqq|z| / \rho^{2} .
$$

The radius of convergence $\rho$ of the Taylor expansion $f(z)$ $\equiv \sum_{n=0}^{\infty} a_{n} z^{n}$ satisfies the equation

$$
\varlimsup_{n \rightarrow \infty}\left|a_{n}\right|^{1 / n}=1 / \rho,
$$

and Lemma 3 yields for $|z|>\rho$,

$$
\varlimsup_{n \rightarrow \infty}\left|P_{n}(z)\right|^{1 / n}=|z| / \rho .
$$

Relations (12) and (13) imply through Lemma $2(|z|>\rho>1)$

$$
\varlimsup_{n \rightarrow \infty}\left|p_{n}(z)\right|^{1 / n}=|z| / \rho>1 \text {. }
$$

Thus the sequence $p_{n}(z)$ cannot be bounded when $|z|>\rho$, hence cannot converge. The theorem is established.

This method of proof is somewhat similar to, but by no means identical with, a proof used in an analogous situation by Faber.* The present proof is valid, the reader may notice, if the

* Journal für Mathematik, vol. 150 (1920), pp. 79-106. 
polynomial $p_{n}(z)$ is defined by interpolation to $f(z)$ not in the $(n+1)$ st roots of unity but in the $(n+1)$ st roots of an arbitrary number $\alpha_{n},\left|\alpha_{n}\right| \leqq 1$.

Lemma 3 extends at once to the case of series of form

$$
\sum_{n=0}^{\infty} a_{n} q_{n}(z),
$$

where $\lim _{n \rightarrow \infty}\left|q_{n}(z)\right|^{1 / n}=q(z)$ exists. For such series, as for the more restricted case of power series, regions of convergence and of divergence can be expressed readily* in terms of $q(z)$ and of $\varlimsup_{n \rightarrow \infty}\left|a_{n}\right|^{1 / n}$. Many comparison series of form (14) found by interpolation exist, for which (as for power series) $\varlimsup_{\lim _{n \rightarrow \infty}}\left|a_{n}\right|^{1 / n}$ can be determined at once from the singularities of the function expanded; such a comparison series may be a series of interpolation, and may be an expansion in polynomials which belong to a more or less arbitrary region. $\dagger$ Whenever such a comparison series is given, the method of proof of the theorem of the present note yields the divergence of suitable "neighboring" sequences of polynomials of interpolation. Thus the present method has a much wider application than the italicized theorem suggests, by the use no longer of power series but of more general comparison series.

But if an arbitrary sequence of polynomials of interpolation is given (for instance, converging to the defining function maximally on a given point set $\ddagger$ ), it may be difficult to determine a suitable comparison series (14) of the kind required for application of the present method. Thus there still remain many sequences of polynomials of interpolation for which exact regions of uniform convergence are known but whose divergence at all points exterior to those regions has never been established; still other methods need to be devised for the study of such divergence.

HARVARD UNIVERSITY

* See op. cit., §3.4, Theorem 5.

$\dagger$ For instance, op. cit., Chapter 7, especially $\$ 7.2$, Corollary 4 to Theorem 2. $\ddagger$ See op. cit., Chapter 7. 